\title{
GENERIC BIFURCATION OF PERIODIC POINTS
}

\author{
BY \\ K. R. MEYER( $\left.{ }^{1}\right)$
}

\begin{abstract}
This paper discusses the bifurcation of periodic points of a generic symplectic diffeomorphism of a two manifold that depends on a parameter. A complete classification of the types of bifurcation that can occur in the generic case is given.
\end{abstract}

Introduction. This paper discusses the bifurcation of periodic points of an area preserving diffeomorphism that depends on a parameter. In the spirit of Thom and Smale only the generic case is considered. As in Smale's work on discrete dynamical systems the present problem was suggested by problems in ordinary differential equations (see [1]). Whereas Smale modeled his theory on differential equations with dissipation the present problem was suggested by conservative differential equations.

Throughout this paper smooth will always mean $C^{\infty}$. Let $M$ be a smooth, compact, two dimensional, symplectic manifold, $S=S^{1}$ the circle considered as a smooth manifold and $\mathscr{F}$ the space of all smooth mappings $\varphi: M \times S \rightarrow M$ with the property that for each $s \in S$ the map $\varphi_{s}=\varphi(\cdot, s): M \rightarrow M$ is a symplectic diffeomorphism. Let $\mathscr{F}$ have the usual topology of $C^{\infty}$ maps. The space $\mathscr{F}$ is complete and therefore is a Baire space. A point $(x, s) \in M \times S$ is called a periodic point of least period $m$ if $\varphi_{s}^{m}(x)=x$ and $m$ is the smallest positive integer for which this holds. Any multiple of $m$ will be called a period of $(x, s)$. If $(x, s)$ is a periodic point of $\varphi$ of least period $m$ then each point $\left(\varphi_{s}^{i}(x), s\right) \in M \times S$ is a distinct periodic point of least period $m$ for $i=1, \ldots, m$ and the set $\bigcup_{i=1}^{m}\left(\varphi_{s}^{i}(x), s\right)$ is called the orbit of $\varphi$ through $(x, s)$.

We wish to discuss the nature of the set of periodic points for $\varphi \in \mathscr{F}$ and in particular the manner in which these periodic points depend on the parameter $s \in S$. Instead of discussing the general element $\varphi \in \mathscr{F}$ we define a subset $\mathscr{G} \subset \mathscr{F}$ and discuss the periodic points for $\varphi \in \mathscr{G}$. Then by showing $\mathscr{G}$ is a residual subset of $\mathscr{F}$ insures that the discussion dealt with the generic case.

The problem discussed here is closely related to the following problems in the theory of differential equations. Consider Duffing's equation $d^{2} x / d t^{2}+x+x^{3}$

Received by the editors July 26, 1969.

AMS Subject Classifications. Primary 3440, 3445, 3451, 3465, 5720, 5750.

Key Words and Phrases. Generic, Baire space, Baire category, symplectic diffeomorphism, periodic points, Hamiltonian differential equations, bifurcation, stability.

( $\left.{ }^{1}\right)$ This research is supported by ONR contract number Nonr 3776(00).

Copyright (C) 1970, American Mathematical Society 
$=E \cos \omega t$ where $x$ is a scalar and $E$ and $\omega$ are positive parameters. Consider the map $T: R^{2} \times R_{+} \times R_{+} \rightarrow R^{2}$ defined by

$$
T(x, y, \omega, E)=\left(\varphi\left(2 \pi \omega^{-1}, x, y, \omega, E\right), \frac{d}{d t} \varphi\left(2 \pi \omega^{-1}, x, y, \omega, E\right)\right)
$$

where $\varphi(t, x, y, \omega, E)$ is the solution of the above equation satisfying the initial conditions $\varphi(0, x, y, \omega, E)=x$ and $\varphi(0, x, y, \omega, E)=y$. It is easy to see that if $(x, y, \omega, E)$ is a periodic point of $T$ of least period $m$ then $\varphi(t, x, y, \omega, E)$ is a periodic solution of the above equation with least period $m 2 \pi / \omega$. Moreover for fixed $\omega$ and $E$ the map $T$ is area preserving. By holding $\omega$ fixed and varying $E$ one studies how the periodic solutions of this equation depend on the amplitude of the forcing term. By holding $E$ fixed and letting $\omega$ vary one studies how the periodic solutions depend on the frequence of the forcing term.

Consider now a Hamiltonian vector field $X$ with Hamiltonian $H$ on the four dimensional symplectic manifold $N$. Let $\Gamma$ be a periodic solution of $X$ with $H(\Gamma)=h_{0}$. One can choose cross sections $\Sigma$ and $\Sigma^{\prime}$ to $\Gamma$ such that (a) $\Sigma \subset \Sigma^{\prime}$, (b) $\Sigma$ and $\Sigma^{\prime}$ are 3 disks, (c) the energy level $H=h$ meets $\Sigma$ and $\Sigma^{\prime}$ in the 2 disks $\sigma(h)$ and $\sigma^{\prime}(h)$ for $h$ near $h_{0}$, (d) the cross section map defined by $\varphi$ carries $\sigma(h)$ into $\sigma^{\prime}(h)$. The cross section map for fixed $h$ is known to be area preserving. Instead of considering $\varphi$ as mapping $\Sigma \rightarrow \Sigma^{\prime}$ carrying $\sigma(h)$ into $\sigma^{\prime}(h)$ we can identify all the $\sigma(h)$ and $\sigma^{\prime}(h)$ with one 2-disk and consider a one parameter family of area preserving diffeomorphisms of the disk.

The local analysis of $\S 1$ is based on the standard methods of bifurcation theory that have been developed by many authors since the time of Poincaré. We have only selected from this vast theory those cases which are generic. Indeed most of the phenomena discussed in $\S 1$ has been or can be verified for the Duffing's equation given above by small parameter methods. The present study was motivated by the results of numerical experiments performed in the restricted, planar three body problem by Deprit and Henrard [2] and Palmore [3]. These studies contain examples of all the forms of bifurcation considered in $\S 1$ plus some others. The reader is referred to these works for a further discussion of bifurcation theory as it applies to a specific example, and for further references.

The global analysis of $\S 2$ is based on the transversality theory developed by Thom and others. The results of Robinson [5] are closely related to the present study. Robinson considered Hamiltonian vector fields on an arbitrary symplectic manifold and has shown that generically periodic solutions lie on smooth twodimensional submanifolds. He has also established that certain irrationality conditions normally used in the study of Hamiltonian systems are generic.

By comparing our formulation and the two examples given above one sees that we have idealized the problem by assuming the basic space $M$ and the parameter space $S$ are compact. In both the above examples the basic and the parameter space are open. The assumption of compactness can be dropped if one uses the Whitney 
topology on $\mathscr{F}$ as was done in the analogous theorem presented in [4]. We have assumed compactness simply to shorten the proofs. Since bifurcation is basically local, the classification of periodic points presented in $\S 1$ remains the same in the noncompact case.

The author would like to thank Dr. Julian Palmore for the many enlightening conversations on the nature of bifurcation in the restricted three body problem. These conversations determined the course of the present study. The author would also like to thank Professor J. Moser for several suggestions and corrections to a preliminary draft of this paper.

1. Local theory. Let $\varphi \in \mathscr{F}$ and $(x, s) \in M \times S$ be a periodic point of $\varphi$ of least period $m$. The eigenvalues of $d \varphi_{s}^{m}: T_{x} M \rightarrow T_{x} M$ are called the multipliers of $(x, s)$. Since $\varphi_{s}$ is a symplectic diffeomorphism the multipliers of $(x, s)$ are of the form $\lambda$ and $\lambda^{-1}$ and so either lie on the unit circle or on the real line.

Definition 1.1. A periodic point of $\varphi \in \mathscr{F}$ is called hyperbolic (elliptic) if its multipliers $\lambda$ and $\lambda^{-1}$ satisfy $|\lambda| \neq 1$ (resp. $|\lambda|=1$ and $\lambda \neq \pm 1$ ).

Since $\varphi^{m} \in \mathscr{F}$ when $\varphi \in \mathscr{F}$, often it is sufficient to consider only fixed points. The following classical lemma follows easily from the implicit function theorem.

LEMMA 1.2. Let $(x, s)$ be a fixed point of $\varphi \in \mathscr{F}$. If the multipliers of $(x, s)$ are not +1 then there exists neighborhoods $U$ and $V, x \in U \subset M, s \in V \subset S$ and a smooth function $y: V \rightarrow U$ such that $y(s)=x$ and $\{(y(\tau), \tau): \tau \in V\} \subset U \times V$ is the fixed point set of $\varphi$ in $U \times V$.

Thus if the multipliers of the fixed point $(x, s)$ are not +1 then near $(x, s)$ the fixed points of $\varphi$ lie on a smooth one dimensional submanifold of $M \times S$ parametrized by $\tau \in V \subset S$. But if the multipliers of $(x, s)$ are $k$ th roots of unity one might expect periodic points of period $k$ arbitrarily near $(x, s)$.

Extremal periodic points. For the present we shall work locally and suppress the parameter. Let

$$
Q=Q(q, p), \quad P=P(q, p)
$$

define a smooth area preserving diffeomorphism of an open disk centered at $q=p=0$ in the $q, p$ plane into the plane. Following Poincaré [6] one observes that

$$
\Omega=(P-p) d(Q+q)-(Q-q) d(P+p)
$$

is a closed form in $q$ and $p$ and so there exists a generating function $G(q, p)$ such that $d G=\Omega$. Assume (1.3) has a fixed point whose multipliers are not -1 . For simplicity assume this fixed point is at $q=p=0$. Since the multipliers of this fixed point are not -1 one can introduce new variables

$$
\xi=Q+q, \quad \eta=P+p
$$

so that

$$
\partial G / \partial \xi=P-p, \quad \partial G / \partial \eta=q-Q .
$$


Thus a fixed point of (1.3) whose multipliers are not -1 corresponds to a critical point of $G$. Conversely if $G(\xi, \eta)$ is a smooth function with a critical point at $\xi=\eta=0$ with Hessian determinants $\Delta$ not equal to $-1 / 4$ then the equations (1.5) and (1.6) can be solved locally to yield an area preserving diffeomorphism of the form (1.3) with $q=p=0$ as a fixed point.

If the quadratic part of $G$ is given by

$$
G=\frac{1}{2}\left\{\alpha \xi^{2}+2 \beta \xi \eta+\gamma \eta^{2}\right\}
$$

with $4 \Delta=\alpha \gamma-\beta^{2} \neq-1$, then the linear part of (1.3) is given by

$$
\left(\begin{array}{l}
Q \\
P
\end{array}\right)=\frac{1}{1+4 \Delta}\left(\begin{array}{cc}
(1-\beta)^{2}-\alpha \gamma & -2 \gamma \\
2 \alpha & (1+\beta)^{2}-\alpha \gamma
\end{array}\right)\left(\begin{array}{l}
q \\
p
\end{array}\right)
$$

and conversely.

Let $T$ be the trace of the matrix in (1.8) then $T=2(1-4 \Delta)(1+4 \Delta)^{-1}$. Hence a hyperbolic fixed point of (1.3) corresponds to a saddle point of $G$ and an elliptic fixed point of (1.3) corresponds to a maximum or minimum of $G$.

Of course, if the original map (1.3) depends on a parameter then the function $G$ depends on a parameter also.

DeFINITION 1.9. Let $(x, s) \in M \times S$ be a periodic point of least period $m$ of $\varphi \in \mathscr{F}$. Then $(x, s)$ will be called an extremal periodic point if in some local coordinate system: $q, p$ canonical coordinates in $M$ with $q(x)=p(x)=0$ and $\varepsilon$ the coordinate in $S$ with $\varepsilon(s)=0$; the map $\varphi^{m}$ is derived by (1.5) and (1.6) from a generating function $G(q, p, \varepsilon)$ such that

$$
G=\partial G / \partial q=\partial G / \partial p=\partial^{2} G / \partial p^{2}=\partial^{2} G / \partial q \partial p=0
$$

and

$$
\partial^{2} G / \partial q^{2} \neq 0, \quad \partial^{2} G / \partial p \partial \varepsilon \neq 0, \quad \partial^{3} G / \partial p^{3} \neq 0 .
$$

The typical example of such a function is $G=q^{2} / 2+\varepsilon p+p^{3} / 3$.

Proposition 1.10. Let $(x, s) \in M \times S$ be an extremal fixed point of $\varphi \in M \times S$. Then $(x, s)$ lies on a smooth local one parameter family of fixed points of $M \times S$. The point $(x, s)$ divides this local family into two arcs each of which are parameterized by $s \in V \subset S$ and one arc is entirely hyperbolic fixed points and the other arc is entirely elliptic fixed points. Moreover in any coordinate system $\varepsilon: U \subset S \rightarrow R$ the function $\varepsilon$ achieves a nondegenerate maximum or minimum along this family.

Proof. From the preceding remarks it is enough to look at the critical points of the function $G$.

The Jacobian of the equations $G_{p}=G_{q}=0$ at $q=p=\varepsilon=0$ is

$$
\left(\begin{array}{lll}
0 & 0 & G_{p \varepsilon} \\
0 & G_{q q} & G_{\varepsilon q}
\end{array}\right)
$$

which is of rank 2 by assumption. Thus we can find functions $\xi(p)$ and $\eta(p)$ such 
that $G_{p}(p, \xi(p), \eta(p)) \equiv G_{q}(p, \xi(p), \eta(p)) \equiv 0$. From these identities one computes that

$$
\frac{d}{d p} \eta(0)=\frac{d}{d p} \xi(0)=0 \quad \text { and } \quad \frac{d^{2} \eta}{d p^{2}}(0)=-G_{p p p} \mid G_{p \varepsilon} \neq 0
$$

Thus $\eta$ achieves a nondegenerate maximum or minimum at 0 . Using these facts one computes that

$$
\left(\begin{array}{ll}
G_{q q} & G_{p q} \\
G_{p q} & G_{p p}
\end{array}\right)(p, \xi(p), \eta(p))=\left(\begin{array}{cc}
G_{q q}(0) & 0 \\
0 & p G_{p p p(0)}
\end{array}\right)+o(p) .
$$

Thus for $p=0$ the critical point $(p, \xi(p), \eta(p))$ is nondegenerate and is a saddle for $p$ on one side of $p=0$ and a maximum or minimum for $p$ on the other side of zero.

Proposition 1.11. Let $(x, s)$ be an extremal fixed point of $\varphi \in \mathscr{F}$. Then there exists a neighborhood $U, x \in U \subset M$, such that for each $y \in U-\{x\}$ either $\varphi_{s}^{\alpha}(y) \rightarrow x$ as $\alpha \rightarrow \infty$ or for some $\alpha_{0}>0, \varphi_{s}^{\alpha_{0}}(y) \notin U$.

REMARK. Since not all points can approach $x$ under iteration at least one must leave the neighborhood $U$ and thus $x$ is unstable. Since not all points can leave the neighborhood under iteration there must be at least one point $y \in U-\{x\}$ that is asymptotic to $x$, i.e. $\varphi_{s}^{\alpha}(y) \rightarrow x$ as $\alpha \rightarrow \infty$. Moreover the proposition states that $U$ does not contain the orbit of any other periodic point.

Proof. Let $O(n)$ denote a function defined in a neighborhood of the origin in the plane which vanishes along with all its partial derivation up to order $n-1$ at the origin. We may assume that $G$ is of the form

$$
G=\xi^{2} / 2+a \eta^{3} / 3+b \eta^{2} \xi / 2+c \eta \xi^{2}+d \xi^{3}+O(4),
$$

where $a \neq 0$. By the formulas (1.5) and (1.6)

$$
Q=q-4\left\{a(p+q)^{2}+b(p+q) q+c q^{2}\right\}+O(3), \quad P=p+2 q+O(2) .
$$

Consider the function $V=\beta q-q p$ then

$$
\begin{aligned}
\Delta V & =V(Q(q, p), P(q, p))-V(q, p) \\
& =-4 \beta\left\{a(p+q)^{2}+b(p+q) q+c q^{2}\right\}-2 q^{2}+O(3) .
\end{aligned}
$$

If $\beta$ is chosen small enough and with $\beta a$ positive then the quadratic part of $\Delta V$ will be negative definite. Choose a compact neighborhood $N$ of the origin such that $\Delta V$ is negative definite with respect to the origin in all of $N$.

Assume there exists a sequence of points $\left\{\left(q_{i}, p_{i}\right)\right\}$ such that $\left(q_{i}, p_{i}\right) \in N$ for all $i \geqq 1, q_{i+1}=Q\left(q_{i}, p_{i}\right), p_{i+1}=P\left(q_{i}, p_{i}\right)$ for $i \geqq 2$ and $\left(q_{i}, p_{i}\right) \notin N^{\prime}$ where $N^{\prime}$ is an open neighborhood of $q=p=0, N^{\prime} \subset N$. Let $m$ be the minimum of $V$ on $N$ and $\delta$ the minimum of $-\Delta V$ on $N-N^{\prime}$. Note that $\delta>0$. Then

$$
m \leqq V\left(q_{i}, p_{i}\right)=V\left(q_{1}, p_{1}\right)+\sum_{1}^{i-1} \Delta V\left(q_{i}, p_{i}\right) \leqq V\left(q_{1}, p_{1}\right)-(i-1) \delta
$$

for all $i \geqq 2$. But this is clearly impossible since $\delta>0$. 
Transitional periodic point. We now describe the local behavior near a periodic point whose multipliers are -1 .

Definition 1.12. Let $(x, s) \in M \times S$ be a periodic point of $\varphi \in \mathscr{F}$ of least period $m$ and multipliers -1 . The point $(x, s)$ will be called a transitional periodic point if in some coordinate system- $q$ and $p$ coordinates in $M$ with $q(x)=p(x)=0$ and $\varepsilon$ coordinates in $S$ with $\varepsilon(s)=0$ - the map $\varphi^{m}$ has the form

$$
\varphi^{m}:(q, p, \varepsilon) \rightarrow(Q, P), \quad Q=-q+p+O(2), \quad P=-p+O(2),
$$

and $\gamma \neq 0$ and $\alpha^{2}+2 \eta \neq 0$ where

$$
\frac{\partial^{2} P}{\partial q^{2}}=2 \alpha, \quad \frac{\partial^{2} P}{\partial q \partial p}=\beta, \quad \frac{\partial^{2} P}{\partial q \partial \varepsilon}=\gamma, \quad \frac{\partial^{3} P}{\partial q^{3}}=6 \delta, \quad \frac{\partial^{2} Q}{\partial q^{2}}=2 \zeta,
$$

and $\eta=\delta+\alpha \zeta+\alpha \beta / 2$.

Proposition 1.13. Let $(x, s) \in M \times S$ be a transitional fixed point of $\varphi \in \mathscr{F}$. Let $U, V$ and $y$ be as in Lemma 1.2. Then $V$ can be chosen so small that $V-\{s\}$ $=V_{+} \cup V_{-}$where $(y(\tau), \tau), \tau \in V_{+}$is a hyperbolic fixed point of $\varphi$ and $(y(\tau), \tau)$, $\tau \in V_{-}$is an elliptic fixed point of $\varphi$.

Moreover if $V$ is small enough either

(A) there exists smooth functions $z_{1}$ and $z_{2}: V_{+} \rightarrow U$ such that $\left\{\left(z_{1}(\tau), \tau\right)\right.$, $\left.\left(z_{2}(\tau), \tau\right)\right\}, \tau \in S$ is the orbit of an elliptic periodic point of least period 2 and $z_{i}(\tau) \rightarrow x$ as $\tau \rightarrow s$, or

(B) There exists smooth functions $z_{1}$ and $z_{2}: V_{-} \rightarrow U$ such that $\left\{\left(z_{1}(\tau), \tau\right)\right.$, $\left.\left(z_{2}(\tau), \tau\right)\right\}, \tau \in V_{-}$is the orbit of a hyperbolic periodic point of least period 2 and $z_{i}(\tau) \rightarrow x$ as $\tau \rightarrow s$.

REMARK. The proof contains some additional information on the direction and rate of approach of $z_{i}(\tau)$ to $x$.

Proof. Since the determinant of the Jacobian of $\varphi$ is 1 , one can compute that the trace is $-2-\varepsilon \gamma+O\left(\varepsilon^{2}\right)$ and so the first part of the proposition follows.

Let $\gamma\left(\alpha^{2}+2 \eta\right)<0$ and consider the case when $\varepsilon>0$. Make the substitutions $Q=\mu R, P=\mu^{2} T, q=\mu r, p=\mu^{2} t$ where $\mu^{2}=\varepsilon$. (If $\gamma\left(\alpha^{2}+2 \eta\right)>0$ then consider $\varepsilon<0$, let $\mu^{2}=-\varepsilon$ and proceed in the same way.)

Calculate $\varphi:(r, t, \mu) \rightarrow(R, T)$ where

$$
R=-r+\mu\left\{t+\zeta r^{2}\right\}+O\left(\mu^{2}\right), \quad T=-t+\alpha r^{2}+\mu\left\{\gamma r+\beta r t+\delta r^{3}\right\}+O\left(\mu^{2}\right) .
$$

One calculates that the eigenvalues of the Jacobian of this map at the origin are $1 \pm \mu \gamma^{1 / 2}+O(\mu)$ and so the fixed point at the origin is hyperbolic if $\gamma>0$ and elliptic if $\gamma<0$.

From these formulas calculate $\varphi^{2}:(r, t, \mu) \rightarrow\left(R^{2}, T^{2}\right)$ where

$$
R^{2}=r-2 \mu\left\{t-\alpha r^{2} / 2\right\}+O\left(\mu^{2}\right), \quad T^{2}=t-2 \mu\left\{\gamma r+\alpha r t+\eta r^{3}\right\}+O\left(\mu^{2}\right) .
$$

Consider the functions $F(r, t, \mu)=\mu^{-1}\left\{R^{2}-r\right\}$ and $G(r, t, \mu)=\mu^{-1}\left\{T^{2}-t\right\}$. Applying 
the implicit function theorem to $F$ and $G$ one finds there exists functions $r_{i}(\mu)$, $t_{i}(\mu)$ such that $F\left(r_{i}(\mu), t_{i}(\mu), \mu\right) \equiv 0, G\left(r_{i}(\mu), t_{i}(\mu), \mu\right) \equiv 0, i=1,2$. Moreover $t_{i}(0)$ $=-\alpha \gamma /\left(\alpha^{2}+2 \eta\right)$ and $r_{1}(0)=\left(-2 \gamma /\left(\alpha^{2}+2 \eta\right)\right)^{1 / 2}, r_{2}(0)=-\left(-2 \gamma /\left(\alpha^{2}+2 \eta\right)\right)^{1 / 2}$. The functions $z_{i}$ in the coordinates $q, p, \varepsilon$ are $z_{i}: \varepsilon \rightarrow\left(\varepsilon^{1 / 2} r_{i}\left(\varepsilon^{1 / 2}\right), \varepsilon t_{i}\left(\varepsilon^{1 / 2}\right)\right)$ for $\varepsilon>0$.

The eigenvalues of the Jacobian of $R^{2}, T^{2}$ with respect to $r$ and $t$ at these solutions are $1 \pm 2 \mu(-\gamma)^{1 / 2}+O\left(\mu^{2}\right)$. Hence if $\gamma<0$ they are hyperbolic and if $\gamma>0$ they are elliptic.

Bifurcation points. Now we consider periodic points whose multipliers are $k$ th roots of unity. By the Birkhoff normalization procedure [7] one has

LEMMA 1.14. Let $(x, s) \in M \times S$ be a fixed point of $\varphi \in \mathscr{F}$ whose multipliers are $\exp ( \pm 2 \pi l i \mid k)$ where $l$ and $k$ are relatively prime integers, $k \geqq 3,0<l<k$. Then there exists a symplectic chart $(q, p)$ in $M, q(x)=p(x)=0$ and a chart $\varepsilon$ in $S, \varepsilon(s)=0$ such that $\varphi:(q, p, \varepsilon) \rightarrow(Q, P)$ where

$$
\begin{gathered}
q=r^{1 / 2} \cos \theta, \quad p=r^{1 / 2} \sin \theta, \\
Q=R^{1 / 2} \cos \Theta, \quad P=R^{1 / 2} \sin \Theta, \\
\Theta=\theta+2 \pi l / k+\varepsilon \alpha(\varepsilon) / k+\Sigma_{1}^{[(k-2) / 2]}\left\{\beta_{j}(\varepsilon) / k\right\} r^{j}+\{\gamma(\varepsilon) / k\}\{\cos k \theta\} r^{(k-2) / 2}+\Theta_{1}(\theta, r, \varepsilon) \\
R=r+\{2 \gamma(\varepsilon) / k\}\{\sin k \theta\} r^{k / 2}+R_{1}(\theta, r, \varepsilon)
\end{gathered}
$$

and $R_{1}, \Theta_{1}$, are smooth functions of $\theta, r^{1 / 2}=\rho, \varepsilon$ and $2 \pi$ periodic in $\theta$ with

$$
\begin{aligned}
& \frac{\partial^{j} R_{1}}{\partial \rho^{j}}(\theta, 0,0)=0, \quad j=0,1,2, \ldots, k+1 \\
& \frac{\partial^{i} \Theta_{1}}{\partial \rho^{i}}(\theta, 0,0)=0, \quad i=0,1,2, \ldots, k-1 .
\end{aligned}
$$

Definition 1.15. A periodic point $(x, s) \in M \times S$ of least period $m$ of $\varphi \in \mathscr{F}$ whose multipliers are $k$ th roots of unity will be called a $k$-bifurcation periodic point if in the chart described in Lemma 1.14 for $\varphi^{m}, \alpha$ and $\gamma$ are not zero when $k=3 ; \alpha, \gamma$ and $\beta \pm \gamma$ are not zero when $k=4$ and $\alpha, \beta$ and $\gamma$ are not zero when $k>5$. Here and in what follows we shall write $\alpha, \beta, \gamma$ for $\alpha(0), \beta_{1}(0)$ and $\gamma(0)$ respectively.

Proposition 1.16. Let $(x, s) \in M \times S$ be a 3-bifurcation point. Let $U$ and $V$ be as in Lemma 1.2. If $V$ is small enough there exist smooth functions $w_{i}, i=1,2,3$, from $V-\{s\}$ into $U$ such that $\bigcup_{1}^{3}\left(w_{i}(\tau), \tau\right), \tau \in V-\{s\}$ is the orbit of a hyperbolic periodic point of least period 3. Moreover $w_{i}(\tau) \rightarrow x$ as $\tau \rightarrow s$.

REMARK. More detailed information on the rate and direction of approach of $w_{i}$ to $x$ as $\tau$ tends to $s$ can be gleaned from the proof.

Proof. Consider the chart $(x, s)$ as given in Lemma 1.14. Replace $r$ and $R$ by $\varepsilon^{2} r$ and $\varepsilon^{2} R$ respectively and compute $\varphi^{3}:(\theta, r, \varepsilon) \rightarrow\left(\Theta^{3}, R^{3}\right)$, where

$$
\Theta^{3}=\theta+\varepsilon\left\{\alpha+\gamma(\cos 3 \theta) r^{1 / 2}\right\}+O\left(\varepsilon^{2}\right), \quad R^{3}=r+\varepsilon\left\{2 \gamma(\sin 3 \theta) r^{3 / 2}\right\}+O\left(\varepsilon^{2}\right) .
$$


Let $\alpha \gamma>0$ (resp. $\alpha \gamma<0$ ) then by the implicit function theorem there exist functions $\xi_{i}(\varepsilon), \eta_{i}(\varepsilon), i=1,2,3$ such that $\varepsilon^{-1}\left\{\Theta^{3}\left(\xi_{i}(\varepsilon), \eta_{i}(\varepsilon), \varepsilon\right)-\xi_{i}(\varepsilon)\right\} \equiv 0$ and

$$
\varepsilon^{-1}\left\{R^{3}\left(\xi_{i}(\xi), \eta_{i}(\varepsilon), \varepsilon\right)-\eta_{i}(\varepsilon)\right\} \equiv 0
$$

and $\xi_{i}(0)=\pi / 3+2 \pi i / 3\left(\right.$ resp. $\left.\xi_{i}(0)=2 \pi i / 3\right)$ and $\eta_{i}(0)=\alpha / \gamma\left(\right.$ resp. $\left.\eta_{i}(0)=-\alpha / \gamma\right)$.

The functions $w_{i}$ in the original coordinates (i.e. before $r$ and $R$ are replaced by $\varepsilon^{2} r$ and $\left.\varepsilon^{2} R\right)$ are $w_{i}:(\theta, r, \varepsilon) \rightarrow\left(\xi_{i}(\varepsilon), \varepsilon^{2} \eta_{i}(\varepsilon)\right)$. Compute

$$
\frac{\partial\left(\Theta^{3}, R^{3}\right)}{\partial(\theta, r)}=\left(\begin{array}{ll}
1 & 0 \\
0 & 1
\end{array}\right)+\varepsilon\left(\begin{array}{cc}
0 & \mp \frac{1}{2} \gamma( \pm \alpha / \gamma)^{-1 / 2} \\
\mp 6 \gamma( \pm \alpha / \gamma)^{3 / 2} & 0
\end{array}\right)+O\left(\varepsilon^{2}\right) .
$$

Hence the eigenvalues are $1 \pm \varepsilon(3|\alpha \gamma|)^{1 / 2}+O\left(\varepsilon^{2}\right)$ and the fixed points are hyperbolic.

Proposition 1.17. Let $(x, s) \in M \times S$ be a 4-bifurcation fixed point of $\varphi \in \mathscr{F}$ and let $U$ and $V$ be as in Lemma 1.2. If $V$ is small enough either (A) there exists smooth functions $w_{i}, i=1,2,3,4$, from $V-\{s\}$ into $U$ such that $\bigcup_{1}^{4}\left(w_{i},(\tau), \tau\right) ; \tau \in V-\{s\}$, is the orbit of a hyperbolic periodic point of least period 4 and $w_{i}(\tau) \rightarrow x$ as $\tau \rightarrow s$ or (B) there exist smooth functions $z_{i}$ and $w_{i}, i=1,2,3,4$, from $V_{+}$into $U$ such that $\bigcup_{1}^{4}\left(w_{i}(\tau), \tau\right)$ is the orbit of a hyperbolic periodic fixed point of least period 4 and $\bigcup_{1}^{4}\left(z_{i}(\tau), \tau\right)$ is the orbit of an elliptic periodic point of least period 4. Moreover $z_{i}(\tau) \rightarrow x$ and $w_{i}(\tau) \rightarrow x$ as $\tau \rightarrow s$. The set $V_{+} \subset V$ is one of the two connected arcs of $V-\{s\}$.

Proof. The two cases are as (A) $|\gamma|>|\beta|$ or (B) $|\gamma|<|\beta|$. The proof of case (A) is similar to the proof of the previous proposition, and the proof of case (B) is similar to the proof of the next proposition. Therefore we shall leave this proof to the reader and continue to the next proposition.

Proposition 1.18. Let $(x, s) \in M \times S$ be a $k$-bifurcation, $k \geqq 5$, fixed point of $\varphi \in \mathscr{F}$. Let $U$ and $V$ be as in Lemma 1.2 and let $V-\{s\}=V_{+} \cup V_{-}$where $V_{+}$and $V_{-}$ are connected open arcs. Then if $V$ is small enough there exists smooth functions $z_{i}$ and $w_{i}, i=1,2, \ldots, k$ from $V_{+}\left(\right.$or $\left.V_{-}\right)$into $U$ such that $\bigcup_{1}^{4}\left(z_{i}(\tau), \tau\right)$ is the orbit of an elliptic periodic point of least period $k$ and $\bigcup_{1}^{k}\left(w_{i}(\tau), \tau\right)$ is the orbit of a hyperbolic periodic point of least period $k$. Moreover $w_{i}(\tau)$ and $z_{i}(\tau)$ approach $x$ as $\tau$ approaches $s$.

REMARK. More detailed information on the rate and direction of approach of $w_{i}$ and $z_{i}$ to $x$ as $\tau$ approaches $s$ can be gleaned from the proof.

Proof. For definiteness assume $\alpha \beta<0$ - the case when $\alpha \beta>0$ is treated in a similar manner. Compute $\varphi^{k}$ and make the substitution $r \rightarrow \varepsilon r$ for $\varepsilon>0$. Then $\varphi^{k}:(\theta, r, \varepsilon) \rightarrow\left(\Theta^{k}, R^{k}\right)$,

$$
\begin{aligned}
& \Theta^{k}(\theta, r, \varepsilon)=\theta+\varepsilon\{\alpha+\beta r\}+O\left(\varepsilon^{3 / 2}\right), \\
& R^{k}(\theta, r, \varepsilon)=r+\varepsilon^{(k-2) / 2}(2 \gamma \sin k \theta) r^{k / 2}+O\left(\varepsilon^{(k-1) / 2}\right) .
\end{aligned}
$$


Since $k>4$ both $\Theta^{k}$ and $R^{k}$ are at least $C^{1}$ in $r$. Since $\alpha \beta<0$ the implicit function theorem gives the existence of a function $\zeta(\theta, \varepsilon)$ such that

$$
\zeta(\theta, 0)=-\alpha \beta^{-1}>0 \text { and } \Theta^{k}(\theta, \zeta(\theta, \varepsilon), \varepsilon)-\theta \equiv 0 .
$$

Since $\gamma \neq 0$ the implicit function theorem yields the existence of $2 k$ functions $\xi_{i}(\varepsilon)$ and $\eta_{i}(\varepsilon), i=1,2, \ldots, k$ such that $\xi_{i}(0)=2 i \pi / k$ and $\eta_{i}(0)=(2 i+1) \pi / k$ and

$$
R^{k}\left(\xi_{i}(\varepsilon), \zeta\left(\xi_{i}(\varepsilon), \varepsilon\right), \varepsilon\right)-\zeta\left(\xi_{i}(\varepsilon), \varepsilon\right) \equiv 0
$$

and

$$
R^{k}\left(\eta_{i}(\varepsilon), \zeta\left(\eta_{i}(\varepsilon), \varepsilon\right), \varepsilon\right)-\zeta\left(\eta_{i}(\varepsilon), \varepsilon\right) \equiv 0 .
$$

In this coordinate system the fixed points of $\varphi^{k}$ are $\left(\xi_{i}(\varepsilon), \zeta\left(\xi_{i}(\varepsilon), \varepsilon\right), \varepsilon\right)$ and $\left(\eta_{i}(\varepsilon), \zeta\left(\eta_{i}(\varepsilon), \varepsilon\right), \varepsilon\right)$.

We compute the Jacobian $J$ of $\varphi^{k}$ at these fixed points to be

$$
J=\left(\begin{array}{cc}
1+O\left(\varepsilon^{(k-1) / 2}\right) & \varepsilon \beta+O\left(\varepsilon^{3 / 2}\right) \\
\pm \varepsilon^{(k-2) / 2} 2 k \gamma\left(-\alpha \beta^{-1}\right)^{k / 2}+O\left(\varepsilon^{(k-1) / 2}\right) & 1+O\left(\varepsilon^{(k-1) / 2}\right)
\end{array}\right)
$$

where the + sign is to be taken for the fixed points at the angles $\xi_{i}(\varepsilon)$ and the sign is to be taken for the fixed points at the angles $\eta_{i}(\varepsilon)$. One sees that the trace of $J$ is equal to 2 to a very high order in $\varepsilon$. But if one uses the fact that det $J \equiv 1$ one can easily compute that trace $J=2 \mp 2 \varepsilon^{k / 2} \gamma \beta k\left(-\alpha \beta^{-1}\right)^{k / 2}$ and so one set of fixed points are hyperbolic and the other elliptic.

2. The global approximation theorem. In this section the main theorem (2.2) is stated and proved.

Definition 2.1. Let $\mathscr{G}$ denote the set of $\varphi \in \mathscr{F}$ such that (a) each periodic point of $\varphi$ is either elliptic, hyperbolic, extremal or transitional, and (b) if the multipliers of a periodic point $(x, s) \in M \times S$ of $\varphi$ are $k$ th roots of unity, $k \geqq 3$, then $(x, s)$ is a $k$-bifurcation periodic point.

THEOREM 2.2. $\mathscr{G}$ is residual in $\mathscr{F}$.

Thus the periodic points discussed in $\S 1$ are the only generic periodic points. The rest of this section is devoted to proving Theorem 2.2.

Lemma 2.3. Let $(x, s) \in M \times S$ be a fixed point of $\varphi \in \mathscr{F}$. Then there exists a neighborhood $U$ of $(x, s)$ such that for each neighborhood $\mathscr{U}$ of $\varphi$ there is a $\psi \in \mathscr{U}$ and $\psi$ has only extremal periodic points or periodic points whose multipliers are not +1 . Moreover for each such $\psi$ there exists a neighborhood $\mathscr{V}$ of $\psi$ such that all $\zeta \in \mathscr{V}$ have periodic points in $U$ whose multipliers are not +1 or are extremal.

Proof. If the multipliers of $(x, s)$ are not +1 then the lemma follows easily from the implicit function theorem. Let the multipliers of $(x, s)$ be +1 . Let $U^{\prime}$ be a neighborhood of $(x, s)$ and $(q, p, \varepsilon): U^{\prime} \rightarrow R^{3}$ be a chart at $(x, s)$ with $q(x)$ $=p(x)=\varepsilon(s)=0$. Let $U^{\prime}$ be chosen so small that in this coordinate system $\varphi$ takes the form $\varphi:(q, p, \varepsilon) \rightarrow(Q, P)$ where $Q$ and $P$ are given by the formula (1.5) and 
(1.6) and $G$ is defined on all of $U^{\prime}$. That $\varphi$ can be obtained from a generating function $G$ follows from the implicit function theorem and the linear analysis of $\S 1$. Let $U$ and $U^{\prime \prime}$ be open sets containing $q=p=\varepsilon=0$ and such that closure of $U \subset U^{\prime \prime}$ and closure $U^{\prime \prime} \subset U^{\prime}$ and let $\alpha$ be a smooth function which is identically one on $U$ and zero outside $U^{\prime \prime}$. Let $L\left(U^{\prime}, R^{i}\right)$ be the space of smooth maps from $U^{\prime}$ into $R^{i}$ with the Whitney topology.

There exists a neighborhood $\mathscr{W}$ of $G$ such that for all $H \in \mathscr{W}$ the function $\alpha H+(1-\alpha) G$ defines a smooth area preserving diffeomorphism defined on all of $U^{\prime \prime}$ by the formulas (1.5) and (1.6).

The lemma follows (as given below) by applying the jet transversality theorem of Thom to show that there is an open and dense set in $L\left(U^{\prime}, R\right)$ whose critical points with respect to $q$ and $p$ are either nondegenerate or of the form described in Definition 1.9.

If $H \in L\left(U^{\prime}, R\right)$ consider the function $F \in L\left(U^{\prime}, R^{2}\right), F: U^{\prime} \rightarrow R^{2}:(q, p, \varepsilon) \rightarrow$ $(H(q, p, \varepsilon), \varepsilon)$. Observe that a point is a singular point of $F$-i.e. a point where the rank of $F$ is less than 2-if and only if it is a critical point of $H$ with respect to $q$ and $p$, i.e. $\partial H / \partial q=\partial H / \partial p=0$.

In the rest of this lemma we shall use the notation of Levine [8], [9]. Let $J^{j}\left(U^{\prime}, R^{2}\right)$ denote the space of all $j$-jets from $U^{\prime}$ to $R^{2}$. Let $S_{1}^{j}\left(U^{\prime}, R^{2}\right)$ be the regular submanifold of $J^{j}\left(U^{\prime}, R^{2}\right)$ as defined in [9]. In particular $S_{1}^{1}\left(U^{\prime}, R^{2}\right)$ denotes the set of all 1-jets in $J^{1}\left(U^{\prime}, R^{2}\right)$ whose Jacobian has rank identically equal to one. By the jet transversality theorem there is an open and dense set $K \subset L\left(U^{\prime}, R^{2}\right)$ such that if $F_{1} \in K$ then the one jet of $F_{1}$ meets $S_{1}^{1}\left(U^{\prime}, R^{2}\right)$ transversally. Hence there is an $F_{1} \in K$ arbitrarily close to $F$. Since $F$ is of the form $F(q, p, \varepsilon)=(H(q, p, \varepsilon), \varepsilon)$ the function $F_{1}$ will be of the form $\left(H_{1}(q, p, \varepsilon), \varepsilon+\delta(q, p, \varepsilon)\right)$ where $H_{1}$ is close to $H$ and $\delta$ is small. Define $\mu=\varepsilon+\delta(q, p, \varepsilon)$ and so $F_{1}=\left(H_{2}(q, p, \mu), \mu\right)$. Again $H_{2}$ is close to $H$. Since $F_{1} \in K$ at each point where $\partial F_{1} / \partial q=\partial F_{1} / \partial p=0$ one has

$$
\operatorname{rank}\left(\begin{array}{ccc}
\partial^{2} H_{2} / \partial q^{2} & \partial^{2} H_{2} / \partial q \partial p & \partial^{2} H_{2} / \partial q \partial \mu \\
\partial^{2} H_{2} / \partial p \partial q & \partial^{2} H_{2} / \partial p^{2} & \partial^{2} H_{2} / \partial p \partial \mu
\end{array}\right)=2 .
$$

Moreover the set of such points is either empty or a smooth one-dimensional submanifold of $U^{\prime}$.

Levine has shown [8], [9] that there is a regular submanifold $S_{1}^{2}\left(U^{\prime}, R^{2}\right)$ of $J^{2}\left(U^{\prime}, R^{2}\right)$ which has the following property. Let the 1-jet of $F_{1}$ meet $S_{1}^{1}\left(U^{\prime}, R^{2}\right)$ transversally; then the singular points of $F_{1}$ consist of a regular submanifold $D \subset U^{\prime}$. Then the 2-jet of $F_{1}$ meets $S_{1}^{2}\left(U^{\prime}, R^{2}\right)$ transversally if and only if the one jet of $F_{1} / D$ meets $S_{1}^{1}\left(D, R^{2}\right)$ transversally. Thus as before, we can make a small change in $F_{1}$ to $F_{2}$ so that the 2-jet of $F_{2}$ meets $S_{1}^{2}\left(U^{\prime}, R^{2}\right)$ transversally and again by changing the parameter $F_{2}$ can be written in the form $\left(H_{3}(q, p, \mu), \mu\right)$ where $H_{3}$ is close to $H$. By the same type of computation as is found in Proposition 1.10 one finds that the critical points of $H_{3}$ with respect to $q$ and $p$ are either nondegenerate or of the type describing an extremal fixed point. 
The topology of $L\left(U^{\prime}, R^{2}\right)$ is the $C^{\infty}$ Whitney topology since $U^{\prime}$ is open. Since $U^{\prime \prime}$ is interior to $U^{\prime}$ one sees that the supremum topology on functions from $U^{\prime \prime}$ to $R^{2}$ has an open and dense set of functions with the desired type of critical points.

LEMMA 2.4. Let $l$ be a positive integer and $(x, s) \in M \times S$ be a fixed point of $\varphi \in \mathscr{F}$. Then there exisı a neighborhood $U$ of $(x, s)$ such that for each neighborhood $\mathscr{U}$ of $\varphi$ there is $a \psi \in \mathscr{U}$ and (a) each fixed point of $\psi$ in $U$ is either hyperbolic, elliptic, extremal or transitional, and (b) each fixed point of $\psi$ in $U$ whose multipliers are kth roots of unity, $3 \leqq k \leqq l$, are $k$-bifurcation fixed points. Moreover for each such $\psi$ there is a neighborhood $\mathscr{V}$ of $\psi$ such that for all $\zeta \in \mathscr{V}$ the fixed points of $\zeta$ in $U$ satisfy (a) and (b) above.

Proof. In the two-dimensional case an area preserving diffeomorphism can always be obtained locally from a generating function (see [10, pp. 69-70]). Thus perturbations can be made locally and then smoothed out by a bump function as in the previous lemma.

The previous lemma covers the case when the multipliers of $(x, s)$ are +1 since there exists a neighborhood $U$ of an extremal fixed point such that all fixed points of $\varphi$ in $U$ have multipliers close to +1 . Thus we may assume the multipliers of $(x, s)$ are not +1 . By Lemma 1.2 the fixed point $(x, s)$ lies in a one parameter family of fixed points parameterized locally by $\tau \in S$. Hence one can find charts $u: U^{\prime} \rightarrow R^{2}$, $x \in U^{\prime} \subset M, \varepsilon: V^{\prime} \rightarrow R, s \in V \subset S$, such that the fixed points of $\varphi$ in $U^{\prime} \times V^{\prime}$ are given by $u^{-1}(0) \times V^{\prime}$. In local coordinates $\varphi$ has the form $\varphi(u, \varepsilon)=A(\varepsilon) u+h(u, \varepsilon)$ where $h(0, \varepsilon) \equiv 0, \partial h(0, \varepsilon) / \partial u \equiv 0$, and $A(\varepsilon)$ is a smooth curve in $S L(2, R)$. Here $S L(2, R)$ is the special linear group of $2 \times 2$ matrices over the reals or equivalently the group of $2 \times 2$ symplectic matrices.

The set of matrices $B \in S L(2, R)$ whose eigenvalues are $\lambda$ and $\lambda^{-1}$ is given by the smooth two-dimensional submanifold of matrices $B \in S L(2, R)$ such that trace $B=\lambda+\lambda^{-1}$. Let $N$ be the smooth submanifold of $S L(2, R)$ given by those matrices whose eigenvalues are $k$ th roots of unity $2 \leqq k \leqq l$. By the elementary transversality theorem there is a smooth curve $C: V \rightarrow S L(2, R)$ arbitrarily close to $A$ such that $C$ is transversal to $N$.

The map $\varphi$ can be obtained from a generating function $S$ (see [10] for details). Make a small change in $S$ to obtain a new generating function $\tilde{S}$ such that the map $\zeta$ obtained from $\tilde{S}$ has the form $\zeta(u, \varepsilon)=C(\varepsilon) u+\tilde{h}(u, \varepsilon)$. The fixed points of $\zeta$ whose multipliers are $k$ th roots of unity, $2 \leqq k \leqq l$, are isolated in $U \times V^{\prime}$. Thus we can work locally at each of these fixed points.

Let $\left(u_{0}, \varepsilon_{0}\right) \in U^{\prime} \times V^{\prime}$ be a fixed point of $\zeta$ whose multipliers are $k$ th roots of unity $2 \leqq k \leqq l$. There exists a neighborhood $W$ of $\left(u_{0}, \varepsilon_{0}\right)$ which contains no other fixed points whose multipliers are $j$ th roots of unity $2 \leqq j \leqq l$ and a function $g(u, \varepsilon)$ arbitrarily close to $h(u, \varepsilon)$ on $W$ such that $\left(u_{0}, \varepsilon_{0}\right)$ is a transitional fixed point of $\psi=C(\varepsilon) u+g(u, \varepsilon)$ if $k=2$ or is a $k$-bifurcation fixed point of $\psi=C(\varepsilon) u+g(u, \varepsilon)$ if $2<k \leqq l$. 
The map $\psi$ can be patched together using generating functions as was done in Lemma 2.3. The second part of the lemma, i.e. openness, is obvious.

REMARK 2.5. In Lemmas 2.3 and 2.4 we could assume that $(x, s)$ is a periodic point of least period $h$ and arrive at the same conclusion. Since if $(x, s)$ is a periodic point of least period $h$ there is a neighborhood $U$ of $x$ and a neighborhood $V$ of $s$ such that the sets $W^{j}=\left\{\varphi_{s}^{j}(U): s \in V\right\}$ are disjoint for $0 \leqq j<h$. Thus the small perturbation of $\varphi^{h}$ can be made by a small perturbation of $\varphi$ on $W^{h-1} \times V \rightarrow W^{h}$ without effecting the value of $\varphi$ on $W^{j} \times V, 0 \leqq j \leqq h-2$.

Let $M \times S$ be given some metric. If $\varphi \in \mathscr{F}$ let $K(i, \varphi)$ be the set of all fixed points $(x, s)$ of $\varphi^{i}$ and let $L(i, \varphi)$ be the subset of $K(i, \varphi)$ consisting of all points $(x, s)$ such that $(x, s)$ is a fixed point of $\varphi^{j}$ for $0<j<i$. Clearly $K(i, \varphi)$ and $L(i, \varphi)$ are compact.

Definition 2.6. Let $\mathscr{G}(l, h, \alpha)$ be the set of all $\varphi \in \mathscr{F}$ such that if $(x, s) \in K(i, \varphi)$, $0<i \leqq l$, and the distance of $(x, s)$ to $K(i, \varphi)$ is greater than or equal to $\alpha$ then $(x, s)$ is either hyperbolic, elliptic, extremal or transitional and if $(x, s)$ has multipliers that are $j$ th roots of unity, $2<j \leqq h$, then $(x, s)$ is a $j$-bifurcation periodic point.

Since $\mathscr{G}=\bigcap_{n} \mathscr{G}(n, n, 1 / n)$, Theorem 2.2 follows from:

Lemma 2.7. $\mathscr{G}(l, h, \alpha)$ is open and dense in $\mathscr{F}$.

Proof. We let $l$ and $\alpha$ be fixed and use an induction proof on $h$. As a temporary definition let us call any periodic point an elementary periodic point if it is hyperbolic, elliptic, extremal or transitional and if its multipliers are $j$ th roots of unity $3 \leqq j \leqq l$ then it is a $j$-bifurcation periodic point.

Let $\varphi \in \mathscr{F}$ and a neighborhood $\mathscr{U}$ of $\varphi$ be given. The set $K(1, \varphi)$ is compact and $L(1, \varphi)=\varnothing$. Thus $K(1, \varphi)$ can be covered by a finite number of open sets $U_{m}$, $m=1, \ldots, r$ as given in Lemma 2.4. We can suppose that $\mathscr{U}$ is small enough that for any $\zeta \in \mathscr{U}$ the only fixed points of $\zeta$ lie in $\bigcup_{m=1}^{r} U_{i}$.

Apply Lemma 2.4 to $\varphi$ in $U_{1}$ to obtain a function $\varphi_{1} \in \mathscr{U}$ and a neighborhood $\mathscr{U}_{1} \subset \mathscr{U}$ of $\varphi_{1}$ such that $\varphi_{1}$ and any $\zeta \in \mathscr{U}_{1}$ have only elementary fixed points in $U_{1}$. Repeat this procedure to find functions $\varphi_{i}$ and neighborhoods $\mathscr{U}_{i}$ of $\varphi_{i}$ such that $\mathscr{U}_{i} \subset \mathscr{U}_{i-1} \subset \mathscr{U}$ and $\varphi_{i}$ has only elementary fixed points in $U_{i}$ (and hence in $\bigcup_{m=1}^{i} U_{m}$ ). Then $\varphi_{r}$ is the desired function and $G(l, 1, \alpha)$ is dense in $\mathscr{F}$.

Let $\varphi \in \mathscr{G}(l, 1, \alpha)$. Then $K(1, \varphi)$ is compact and so can be covered by a finite number of open sets $U_{m}, m=1, \ldots, r$ as given in Lemma 2.4. We can choose a neighborhood $\mathscr{U}$ of $\varphi$ such that any $\zeta \in \mathscr{U}$ has all its fixed points in $\bigcup_{m=1}^{r} U_{m}$. By the second part of Lemma 2.4 there exists neighborhoods $\mathscr{V}_{m}$ of $\varphi$ such that for each $\zeta \in \mathscr{V}_{m}$ the only fixed points of $\zeta$ in $U_{m}$ are elementary. $\mathscr{U} \cap\left\{\bigcap_{m=1}^{r} \mathscr{U}_{m}\right\}$ is a neighborhod of $\varphi$ in $\mathscr{G}(l, 1, \alpha)$ and so $\mathscr{G}(l, 1, \alpha)$ is open.

The induction step is the same as the first step since the set of points in $K(h, \alpha)$ whose distance to $L(h, \alpha)$ is greater than or equal to $\alpha$ is a compact set. See also the Remark 2.5.

PostSCRIPT. One might ask to what extent the low dimensionality of $M$ and $S$ 
restricts the nature of bifurcation. As long as the parameter space is one-dimensional it is the author's belief that no essentially new forms of bifurcation will occur if the dimension of $M$ is increased and so hold no further interest.

The general case of a $2 n$ dimensional symplectic manifold $M$ and an $m$ dimensional parameters space would contain too many essentially different forms of bifurcation that the dictionary would be too long to comprehend.

\section{REFERENCES}

1. S. Smale, Differentiable dynamical systems, Bull. Amer. Math. Soc. 73 (1967), 747-817. MR 37 \#3598.

2. A. Deprit and J. Henrard, A manifold of periodic orbits, Advances in Astronomy and Astrophysics 6 (1968).

3. J. Palmore, Bridges and natural centers in the restricted three body problem, University of Minnesota Report, 1969.

4. M. Peixoto, On an approximation theorem of Kupka and Smale, J. Differential Equations 3 (1967), 214-227. MR 35 \#499.

5. C. Robinson, A global approximation theorem for Hamiltonian systems, Proc. Summer Inst. Global Analysis, (to appear).

6. H. Poincaré, Les méthodes nouvelles de la mécanique céleste, Tome 3, Gauthier-Villars, Paris, 1899.

7. G. Birkhoff, Dynamical systems, Amer. Math. Soc. Colloq. Publ., vol. 9, Amer. Math. Soc., Providence, R. I., 1927.

8. H. Levine, Singularities of differentiable mappings. I, Mathematisches Institute der Universität, Bonn, 1959.

9. H. Levine, The singularities, $S_{1}^{q}$, Illinois J. Math. 8 (1964), 152-168. MR 28 \#2560.

10. H. Pollard, Mathematical introduction to celestial mechanics, Prentice-Hall, Englewood Cliffs, N. J., 1966. MR 33 \#5357.

UNIVERSITY OF MINNESOTA, MinneAPolis, Minnesota 55455 